\title{
Carnets
}

Revue électronique d'études françaises de l'APEF

Deuxième série - 15 | 2019

Jules Verne et les pouvoirs de l'imagination

\section{Projet Jules Verne à l'école : le tour du monde}

María-Teresa Barea, María-Lourdes Cadena, Ana-María Claver et MaríaPílar Tresaco

(2) OpenEdition

Journals

Édition électronique

URL: http://journals.openedition.org/carnets/9475

DOI : $10.4000 /$ carnets. 9475

ISSN : 1646-7698

Éditeur

APEF

\section{Référence électronique}

María-Teresa Barea, María-Lourdes Cadena, Ana-María Claver et María-Pílar Tresaco, « Projet Jules Verne à l'école : le tour du monde », Carnets [En ligne], Deuxième série - 15 | 2019, mis en ligne le 31 janvier 2019, consulté le 20 avril 2019. URL : http://journals.openedition.org/carnets/9475 ; DOI : $10.4000 /$ carnets. 9475

Ce document a été généré automatiquement le 20 avril 2019

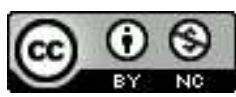

Carnets est mis à disposition selon les termes de la licence Creative Commons - Atribution - Pas d'utilisation commerciale 4.0 International. 


\title{
Projet Jules Verne à l'école : le tour du monde
}

\author{
María-Teresa Barea, María-Lourdes Cadena, Ana-María Claver et María- \\ Pílar Tresaco
}

\section{NOTE DE L'AUTEUR}

T3AxEL, groupe de recherches de l'Université de Saragosse (Espagne). Financement :

Gouvernement d'Aragon-FEDER.

\section{Introduction}

1 Ce qui nous a conduit à la réalisation du projet "Jules Verne à l'école : le tour du monde », c'est le désir d'introduire cet écrivain pour qu'il soit présent dans les classes et que les élèves le connaissent. Nous avons choisi Le tour du monde en quatre-vingt jours parce que c'est un roman dynamique, vivant, optimiste, transversal, qui nous permet de travailler pleinement sur les domaines d'apprentissage à la Maternelle et au Primaire ${ }^{1}$. C'est aussi un roman que les parents connaissent pour l'avoir lu, par les films, la télévision, les dessins animés, les bandes dessinées, entre autres.

Nous tenons à préciser que l'objectif principal n'est pas l'actualisation d'un texte littéraire $d u X x^{e}$ siècle nous permettant de réfléchir sur les problèmes sociaux présents et futurs $^{2}$ qu'il ne s'agit pas d'un projet spécifiquement littéraire, ni d'une mise à jour d'un texte de la littérature française.

Le livre de départ utilisé pour ce projet est l'édition originale publiée par $\mathrm{Hetzel}^{3}$ dont nous avons fait la traduction en adaptant le texte et en respectant ses illustrations, les noms des personnages et le découpage des chapitres. Nous proposons donc aux enseignants des écoles, un texte en espagnol, adapté aux enfants, afin d'être lu en classe, sur lequel des questions et des remarques, proposées au fil de la lecture, permettent à tous les élèves de suivre aisément l'histoire. La lecture et les activités du projet se 
dérouleront tout au long de l'année scolaire, avec une durée variable, une heure ou une heure et demie maximum par semaine. Cependant, ne pouvant pas le présenter dans sa totalité, nous allons expliquer le déroulement des premières séances en Maternelle (classes de 3-6 ans) et pour le Primaire le livre des élèves (classes de 6-8 ans), en guise de bilan du travail réalisé le long de l'année.

\section{L'apprentissage par projet}

L'apprentissage par projet est aussi appelé pédagogie du projet ou pédagogie par projets. Il fait référence à une approche, stratégie ou méthode pédagogique, selon les auteurs, et peut être mené individuellement, en petits groupes ou dans un groupe plus large, voire dans tout l'établissement.

Cette méthode situe les élèves au centre de leurs apprentissages et les encourage à réaliser des tâches qui répondent à des situations réelles. Le projet sera le fil conducteur présent tout au long du processus d'apprentissage, pendant l'année scolaire, qui ne modifie pas le Programme scolaire mais qui s'y intègre (Larmer \& Mergendoller, 2010).

Pour développer un projet dans un établissement, la coordination entre les enseignants et leurs matières et/ou les niveaux est incontournable dans le but de faire travailler tous les élèves sur le même projet : les élèves devront mettre en œuvre les connaissances acquises dans les différentes matières, afin d'en acquérir de nouvelles et de les intégrer dans une structure cohérente qui leur permettra de construire leur apprentissage. Il doit y avoir de préférence un produit final auquel participent tous les élèves, chacun dans la mesure de ses possibilités et selon le niveau, l'âge, les besoins éducatifs, etc.

Cette méthode met en place différentes stratégies d'apprentissage (Proulx, 2008) lui donnant ainsi des avantages significatifs :

- Elle encourage les élèves à apprendre parce que la tâche éveille la curiosité liée à la réalité, ce qui les stimule à faire des recherches. Dans les projets, les participants ne sont pas seulement les élèves et les enseignants, mais aussi des personnes qui n'appartiennent pas à l'école (Motivation).

- Elle développe l'autonomie des enfants puisque ces derniers doivent planifier, distribuer des tâches, prendre des décisions et élaborer un "produit» final (Constructivisme, comme modèle d'apprentissage social).

- Elle favorise l'esprit critique de l'élève en les faisant identifier leurs propres fautes et celles des camarades au sein du groupe, et évaluer leur travail en apprenant des erreurs et par le recours aux connaissances antérieures (Apprentissage significatif).

- Elle renforce les habiletés sociales parce qu'elle permet aux élèves de mettre en commun les idées et d'échanger au sujet du travail qu'ils réalisent. Le travail collaboratif les aide à atteindre l'objectif final (Apprentissage coopératif).

- Elle développe la capacité d'alphabétisation informationnelle des apprenants qui leur permet de chercher, sélectionner et comparer l'information. Les élèves, à l'aide des familles ou autres, devront apporter des informations à la classe (Apprentissage coopératif).

- Elle développe aussi la créativité des enfants et des enseignants, parce qu'elle met en œuvre les stratégies et les idées qui les aident à élaborer le «produit» final. Ce produit peut être très varié (Pédagogie de la créativité).

- Elle répond aux difficultés d'apprentissage à l'école, dues à l'interdépendance entre camarades de classe et aussi aux besoins des étudiants de haut niveau, parce qu'ils peuvent 
aider les autres élèves. De ce fait, le projet suppose la possibilité de développer leurs talents (Accompagnement pédagogique et diversité).

3 Au sein de ce projet nous pouvons travailler sur l'imagination mais il convient que le choix du thème vienne des élèves, quoique guidés par l'enseignant. Pour définir le sujet, on propose une planification correspondant à quelques étapes (Bordalo \& Ginetest, 2006),

- Un remue-méninges avec lequel les enseignants vont extraire les goûts des élèves tout en participant, eux aussi, au choix.

- Une lecture pour éveiller l'intérêt des élèves.

- Une excursion sur le thème ou en relation avec l'écrivain.

- La définition du projet peut se reporter à l'année suivante, afin de donner du temps à la préparation.

- La planification du projet se réalise en fonction de sa temporalité (toutes les matières et les niveaux y participent, par exemple, le vendredi dans un créneau horaire).

Du point de vue méthodologique et de mise en œuvre, un projet doit établir plusieurs paliers pour garantir son développement (RESCOL, 1998/2001) :

- Formation des équipes qui travailleront sur les différents aspects du projet.

- Définition du produit final du projet. Il peut y en avoir plusieurs : brochure, campagne, présentation, recherche scientifique, maquette, pièce de théâtre, etc.

- Planification des tâches prévues dans le projet.

- Recherche des élèves pour bien aboutir à la tâche finale, dans ou hors de l'école.

- Analyse et synthèse afin que les élèves mettent en commun les informations recueillies.

- Élaboration du produit qui se prolonge tout au long de l'année scolaire.

- Présentation du produit, voire de plusieurs produits, en français si c'est possible.

- Évaluation, inter-évaluation et autoévaluation.

Un projet peut se développer dans une école ordinaire ou dans une école d'éducation bilingue. Le projet sur Verne peut aborder tous les domaines d'apprentissage ou matières, compris dans la législation d'éducation espagnole. Il prétend développer les capacités des élèves qui leur permettront d'atteindre les objectifs $d$, e, $g$ et $j$ du Programme d'apprentissage pour la Maternelle, ainsi que les objectifs b et $c$ du Programme pour le Primaire .

Avant d'entamer le projet, il faut attirer l'attention des enfants de façon à ce qu'ils soient motivés pour écouter et découvrir le texte que nous proposons. La motivation est un des fondements de l'apprentissage par projet et elle est à la base de l'apprentissage, en général (Alonso Tapia, 1997).

Il faut être constants et consacrer une séance au projet, tous les vendredis, par exemple, faisant ainsi en sorte que les enfants soient dans l'attente de ce moment. Pour ce, il est important d'établir une routine caractérisée par l'histoire que l'on raconte en classe.

Finalement, nous devrons établir une séquence pédagogique, dégagée de l'histoire, qui en appelle à la participation des enfants. Lorsque nous parlons d'un personnage, nous poserons des questions à son sujet. S'il s'agit d'un pays, nous leur demandons s'ils le connaissent, ou si quelqu'un y est allé et nous le situons sur la carte; pareil pour les animaux. Il est possible d'introduire des changements lors des séances pour que l'improvisation y soit présente, cependant la routine est fondamentale, notamment à la Maternelle. Il faut tenir compte que si le projet se réalise le vendredi, les enfants peuvent croire que les activités du projet se situent hors des tâches scolaires, voire obligatoires, et de ce fait l'enjeu sera de combiner le ludique et le scolaire. 


\section{L'apprentissage avec les textes}

6 La finalité de ce projet est de travailler sur un écrivain français et son œuvre et de contribuer largement à l'alphabétisation des élèves à travers le langage oral et écrit et la littérature. D'après Ana Teberosky, cette alphabétisation n'est pas suffisamment prise en compte à l'école,

El conocimiento sobre las propiedades del lenguaje se adquiere no sólo en la escritura sino también en numerosos intercambios orales, sin embargo la escuela ofrece pocas ocasiones para que los alumnos exhiban ese conocimiento en los comienzos de la escolaridad. (Teberosky, $1990: 2$ ).

7 Le langage se développe dans un but communicatif et, ultérieurement, il devient un moyen d'acquérir des connaissances et de construire et structurer notre pensée, de là l'importance de l'alphabétisation à l'école. Cependant, elle ne commence pas à l'école ; dans une société alphabétisée, les enfants connaissent le langage écrit, non comme résultat d'un enseignement de la lecture et de l'écriture, mais en tant que membres d'une société alphabétisée (Ferreiro \& Teberosky, 2005). Les enfants arrivent à l'école avec des acquis sur la langue et sur les faits de langue, même s'ils ne connaissent pas encore le sens conventionnel de ces concepts. De ce fait, la méthode d'alphabétisation devient un processus interactif dans lequel l'enfant joue un rôle actif, capable de réfléchir et de chercher du sens aux idées et à la pensée (Teberosky \& Ferreiro, 2000).

Dans les classes, les enfants proviennent de différents entourages où la culture écrite occupe une place différente dans leur quotidien. Certains auteurs (Teberosky, 2003) soulignent plusieurs facteurs qui contribuent au développement de l'alphabétisation initiale, tels que les interactions aux niveaux social et affectif entre apprenants et enseignants, les instruments ou les approches didactiques utilisés et le contexte communicatif où s'insère l'apprentissage. Les bienfaits de la littérature en classe sont largement démontrés par de nombreux spécialistes dont certains vont au-delà et présentent les textes classiques pour travailler sur la structure narrative, cependant ce qui nous intéresse pour ce projet, ce sont les activités sur la langue, issues de l'Apprentissage dialogique (Oliver \& Gatt, 2010), qui découlent de la lecture.

Par rapport à l'écriture des enfants, Ana Teberosky et Emilia Ferreiro $(2005: 66)$ distinguent plusieurs étapes dont la première est «l'écriture indifférenciée » où les enfants ne différencient pas les graphies des gribouillis et ils vont faire le même gribouillis pour dessiner que pour écrire. Les premiers dessins des enfants sont considérés une écriture naissante. En Maternelle, il s'agit d'un travail de réécriture et dans l'École élémentaire, de reformuler ou redire.

Un autre aspect important de la littérature est que «tout rapport avec le texte est dans son essence interculturel ( (Séoud, 1997 : 137) et cette approche interculturelle devient de plus en plus importante, aujourd'hui, dans les écoles multiculturelles et favorise une activité de « rencontre vers l'autre » (Abdallah-Prétceille \& Porcher cité par Allam-Idou, 2015 : 104).

\section{Le Projet Jules Verne en Maternelle}

8 Le projet permet de travailler sur les trois domaines d'apprentissage de la Maternelle (3 à 6 ans). Il faut faire le lien entre les contenus du Programme scolaire de la Maternelle en 
Aragon (Espagne) et les tâches issues du roman de Jules Verne.

Nous partirons des activités inspirées de l'histoire du roman et nous chercherons leurs fondements dans l'arrêté concernant la Maternelle cité ci-dessus ${ }^{4}$. Le but de l'éducation en maternelle est de contribuer au développement physique, affectif, social et intellectuel de l'enfant. Toute cette étape se chargera du développement émotionnel et affectif de l'enfant, du mouvement et des habitudes corporelles, des manifestations de communication et de langage, des modes élémentaires de vie commune et de relation sociale, ainsi que de la découverte des caractéristiques physiques et sociales de l'entourage. De plus, cette période favorisera l'élaboration de l'image de soi positive et équilibrée et l'autonomie personnelle.

Cette étape de l'éducation est essentielle dans le parcours des élèves pour garantir leur réussite scolaire. Son rôle est de donner envie aux enfants d'aller à l'école pour apprendre, affirmer et épanouir leurs personnalités. Aussi, selon le Programme scolaire de la Maternelle, dans un contexte de citoyenneté européenne, le développement de la compétence communicative en langue étrangère devient essentiel; c'est pourquoi l'apprentissage de la langue étrangère a été avancé en Maternelle.

9 En Espagne, d'après la Loi de l'Éducation Nationale en vigueur, les enseignements sont organisés en trois domaines d'apprentissage que Jules Verne nous permet d'aborder.

Le premier domaine, Connaissance de soi et autonomie personnelle, s'occupe entre autres des contenus en rapport avec le corps et l'image de soi ainsi qu'avec les activités de la vie quotidienne.

Par rapport à l'image de soi, on peut présenter l'auteur et les personnages du Tour $d u$ monde en quatre-vingt jours. À leur tour, les enfants dessineront les protagonistes, se dessineront eux-mêmes, ainsi que leurs camarades afin de prendre conscience de leurs propres images et de celles des autres.

En relation aux contenus sur la vie quotidienne, les habitudes de Phileas Fogg, toujours invariables, nous permettent de travailler, en classe, sur les routines des enfants, sur le quotidien au sein de leurs familles. Connaître les travaux de Passepartout nous amène à apprendre les différentes professions des parents et des personnes de leurs entourages.

10 Le deuxième domaine d'apprentissage, Connaissance $d u$ monde, favorise la découverte de l'entourage des enfants pour s'y intégrer de la meilleure façon possible. Les contenus sur l'environnement physique, naturel, culturel et de la vie en société prétendent favoriser la découverte et la représentation des différents contextes de l'entourage de l'enfant, ainsi que son intégration. Le voyage nous procure cet apprentissage. Les élèves pourront élaborer une grande affiche pour être exposée à l'école. On y représentera les acquis plus généraux et vastes, comme les pays, les acquis plus concrets et aussi plus petits, les villes, ainsi que les endroits que les héros du roman de Verne traversent ou les paysages qu'ils décrivent.

11 Les éléments de l'entourage seront les différents moyens de transport utilisés par terre ou par mer (train, bateau, traîneau, carriole, tramway, éléphant). Les enfants apporteront des photos pour reconnaître les transports et pour élaborer leurs propres livres. On collera les photos des enfants de la classe, en tant que voyageurs de différents moyens de transport. Ce collage servira à décorer la salle.

De même, l'entrée des personnages dans une pagode favorisera la compréhension de l'interculturel religieux.

Les éléments du paysage parcouru par les protagonistes nous permettent de travailler sur les ports, les villes, les plaines de l'Amérique, la jungle de l'Inde, les montagnes chinoises, 
les torrents, les cascades, les pins, les tunnels, les ponts, les prairies et les lacs. Ce parcours nous permet également d'apprendre la faune de chaque pays : éléphants, bisons, chevaux, oiseaux et loups au sein des histoires, et on en profite pour connaitre les animaux domestiques.

Quant au thème de la culture et la société, nous pouvons travailler sur les habitants de chaque pays : les Anglais, les Américains, les Chinois, les Espagnols, les Japonais, etc.

Le dernier domaine d'apprentissage, Langages: communication et représentation, constitue les instruments d'apprentissage nécessaires pour l'évolution de l'enfant dans son entourage et pour la maitrise des messages de son contexte social.

Ces langages, verbal, audiovisuel, artistique et corporel, sont inclus dans ce domaine.

L'histoire permet à tout moment de poser des questions aux enfants pour exploiter la compétence langagière. Nous pouvons, donc, décrire ou représenter oralement un personnage de l'histoire ou bien lire un paragraphe concret et selon sa description, le dessiner.

L'écoute active de l'histoire permet aux enfants de parler lorsque l'enseignant pose des questions au sujet de ce qu'il lit et de se rapprocher à la littérature à travers l'auteur et son œuvre. Les questions sur chaque personnage favorisent la communication et maintiennent l'attention de l'élève.

Pour aborder la langue écrite, étant donné qu'à la Maternelle, notamment en première année, les rythmes d'apprentissage sont très différents et que certains enfants n'arrivent pas encore à lire, ils devront représenter les lettres de l'alphabet (gribouillis) et les mettre en relation avec des aspects de l'auteur ou de son œuvre.

\section{Déroulement par séances à la Maternelle}

\section{Première séance : L'auteur}

L'enseignant présente Jules Verne en tant qu'écrivain du XIX ${ }^{e}$ siècle et pour ce, au début de chaque séance, il mettra en scène une sorte de rituel au moyen d'une photo de l'auteur, un petit déguisement et une plume d'écrivain, dans le but de motiver les enfants. L'explication de l'enseignant et l'observation des enfants seront essentielles. Ce début sera toujours accompagné d'une musique associée à l'histoire. Aussi, il introduira les matériels du projet : le grand livre de classe Le tour du monde en quatre-vingts jours, où les enfants vont participer et un petit livre individuel qu'ils complèteront, avec des fiches, tout au long de l'année scolaire.

Une fois que l'enseignant s'est déguisé (chemise et plume) pour présenter Jules Verne, les élèves devront, individuellement, remplir la couverture de leurs livres, puis décorer et colorier les plumes et la chemise de Jules Verne.

\section{Deuxième séance : Le monde}

15 Après le rituel de présentation de l'auteur, l'enseignant présente l'histoire du voyage et essaie de faire comprendre aux enfants, au moyen d'un globe terrestre, qu'il est possible de faire le tour du monde et de revenir au même point d'origine, ce qui est d'ailleurs la caractéristique de cette histoire.

Pour le démontrer, il fera faire aux enfants le tour du ballon gonflable, par petit groupe ou deux par deux, se tenant par les mains. Lors de cette activité, l'apprenant profitera 
pour leur demander où se trouvent leurs pays ou bien s'ils en connaissent d'autres. Après ils les situeront sur le ballon.

Ensuite, l'apprenant peut leur proposer de découper le globe terrestre afin de voir tous les pays et, ainsi aplati, le garder dans un livre; c'est alors qu'il présente le planisphère terrestre qui simule le découpage pour finalement leur expliquer que si l'on pouvait le repasser, chacun aurait le sien pour le garder dans son livre (figure 1).

Fig. 1

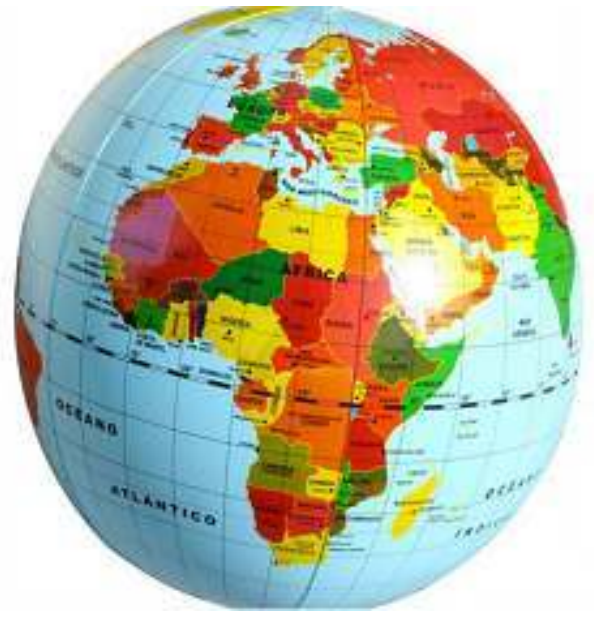

Sinisoidal 6,st Proiection Nas

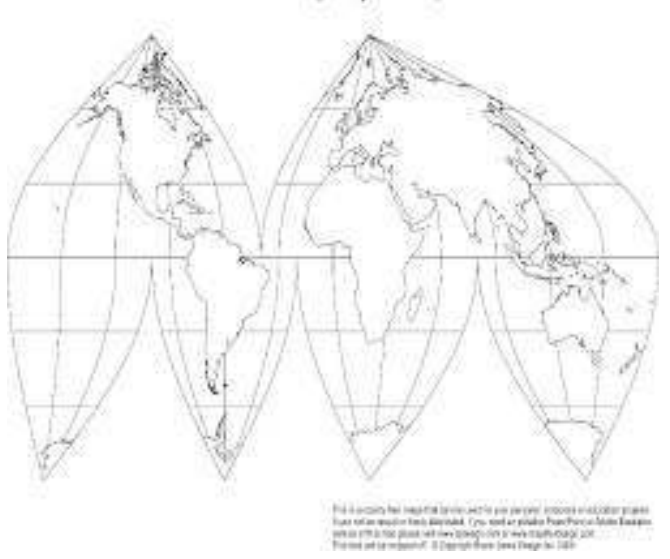

L'enseignant après avoir tracé sur le ballon une ligne imaginaire autour du monde, fera de même sur la fiche du planisphère. Après les élèves devront y retrouver la ville d'Espagne où ils habitent; Paris-France, où habitait Jules Verne ; et Londres-Angleterre, d'où partent les protagonistes voyageurs.

\section{Troisième séance : Phileas Fogg et Passeparpout}

De nouveau, l'enseignant entame le moment de lecture au moyen du rituel. Il présentera les deux protagonistes de l'histoire à travers l'adaptation des illustrations originales du roman de 1872. Tout en lisant à voix haute un passage du récit, il décrira Philéas Fogg, en insistant sur son aspect physique qui correspond à une époque passée (le pantalon, la veste, le chapeau haut-de-forme, les gants, la canne). Il continue le récit et la présentation de Passepartout en tant que domestique de Fogg, puisque celui-ci est très riche. Il expliquera la signification du mot passepartout, qui sert à tout ou qui sait tout faire, et 
finalement on découvrira son aspect physique, différent de Fogg (le pantalon, les bottes, le gilet et surtout la montre avec la petite chaîne).

Après cette première approche, l'enseignant distribuera les images des deux personnages pour que les élèves les colorient et qu'ils entourent les éléments de la description physique (figure 2). Sur la fiche, ils devront aussi identifier et coller, ou écrire le nom des personnages.

Fig. 2
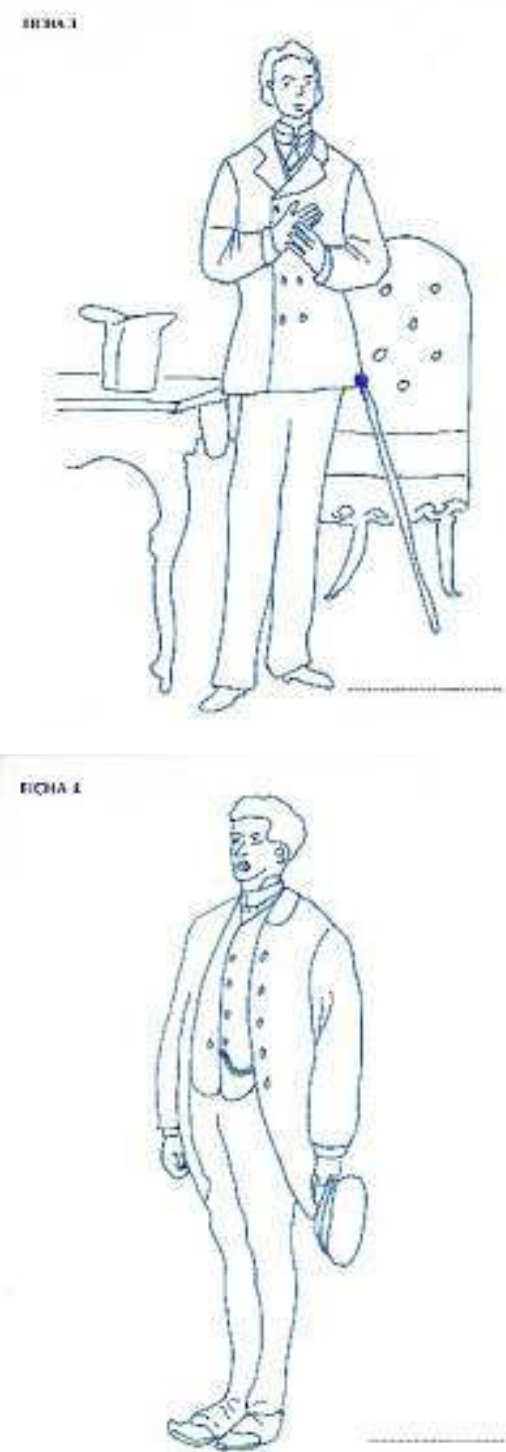

La dernière activité de la séance consistera à identifier, parmi plusieurs types, la montre de Passepartout qui va l'accompagner pendant tout le voyage.

\section{Le Projet Jules Verne au Primaire}

Ce projet permet également de travailler sur les objectifs du Programme scolaire en

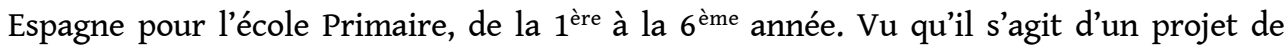
l'école, les enfants entre 6 et 12 ans vont travailler sur Jules Verne dans toutes les 
matières et toutes les classes.

Dans l'étape du Primaire, la réglementation éducative aragonaise nous propose des matières. Il faudra donc tenir compte des contenus et des objectifs de chaque matière pour proposer les tâches de classe. Les projets d'école, selon la législation en Aragon, se développent durant toute l'année scolaire un jour par semaine pendant une heure ou une heure et demie dans tous les cours.

Ce travail propose la mise en œuvre du projet, de façon partielle, pour le premier cycle de l'enseignement primaire, en première et deuxième année, avec trente séances où l'histoire du Tour du monde en quatre-vingts jours sera racontée en même temps que l'on réalise le produit final. Celui-ci consiste en la création, de la part de l'enseignant, d'un grand livre de classe ainsi que des livres individuels sur les voyages de Phileas Fogg.

Pour bien y aboutir, il faudra d'abord attirer l'attention des enfants sur l'auteur, ensuite établir une routine de lecture puis établir les tâches associées au projet. Il est fondamental que les activités découlent toujours de l'histoire, et non au contraire. L'enseignant et les élèves vont élaborer une affiche pour la classe représentant une carte du monde qui leur permettra de suivre le voyage et sur laquelle, chaque jour, à tour de rôle, les élèves marqueront l'itinéraire réalisé par les protagonistes. De même, les enfants colleront des images qu'ils auront apportées. Pour renforcer leurs participations, en fonction des activités prévues sur les apprentissages abordés, ils vont inclure dans leurs livres les noms des continents, des villes, des pays, les transports, les photos des habitants, les animaux. Comme nous avons déjà énoncé en Maternelle, nous pourrons aussi exposer dans la classe un collage photographique représentant les élèves-voyageurs dans le moyen de transport retenu.

Finalement, ils seront en mesure d'élaborer le livre-bilan de Primaire dont nous présentons la couverture (figure 3).

Fig. 3

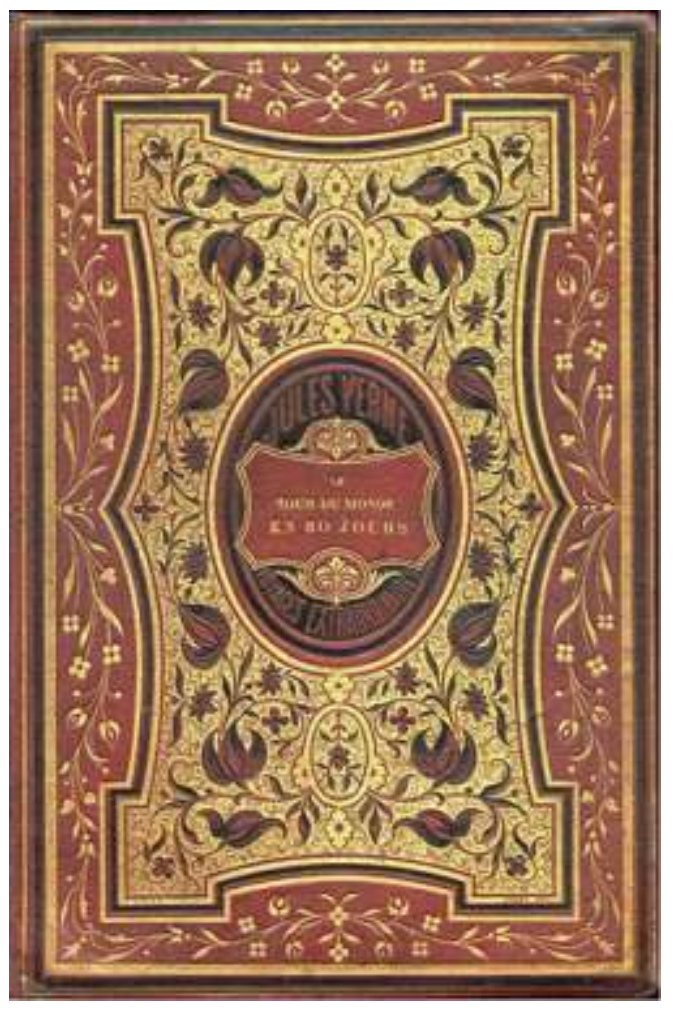




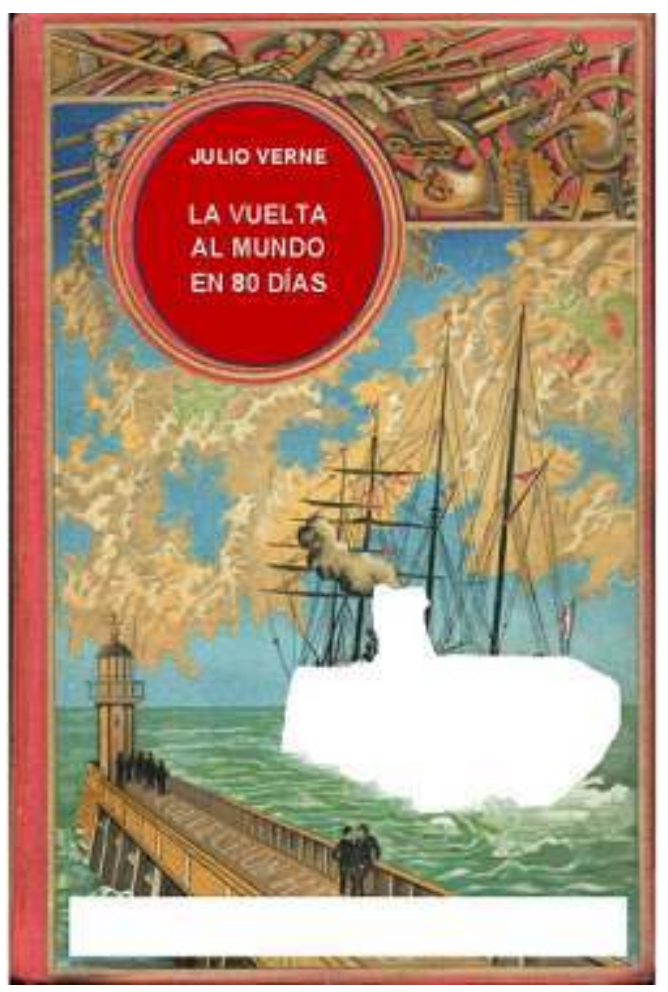

21 Cette approche permet de travailler sur les domaines d'apprentissage de cette étape (6-12 ans) et sur les matières qui constituent l'ensemble des connaissances, compétences, valeurs et attitudes nécessaires pour réussir leur scolarité, leur vie en tant qu'individus et futurs citoyens : Langue espagnole et littérature, Sciences sociales, Enseignement civique et social, Sciences de la nature, Mathématiques, Éducation artistique, Education physique, Première et Seconde langue étrangère.

\section{Langue espagnole et littérature}

Cette matière permet à l'élève d'approfondir progressivement aussi bien sa capacité de compréhension et expression orales et écrites que sa formation littéraire. Les contenus insérés dans cette discipline sont en rapport avec la communication orale et écrite (écouter, parler, lire et écrire), la connaissance de la langue et l'éducation littéraire.

L'élaboration du livre-bilan facilite un travail approfondi sur la langue orale et écrite à travers la biographie de l'auteur, les titres de ses romans, la description et les caractéristiques des personnages ainsi que les aventures du récit. Un exemple de fiche est montré dans la figure 4 . 
Fig. 4

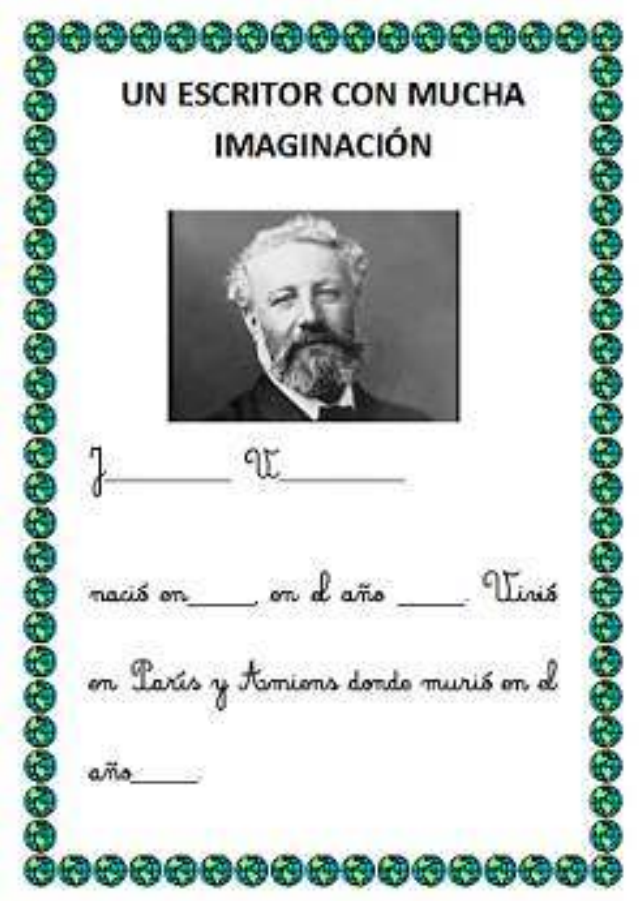

Les noms des villes et des pays seront collés sur la carte du monde. Dans la mesure où les histoires de Verne sont racontées en combinant des circonstances et des incidents qui ne terminent pas à la fin des chapitres, nous profitons de cette caractéristique de la narration pour permettre aux enfants d'inventer la suite du récit, avant de passer au chapitre suivant. Cette activité mobilisent les compétences communicatives orales et écrites.

\section{Sciences sociales, Histoire et Géographie}

Dans cette matière, les contenus sont regroupés en plusieurs rubriques permettant d'identifier les principales unités d'apprentissage des Sciences sociales, telles que les techniques de travail propres à la matière ou l'enseignement par découverte, le monde qui nous entoure (l'Univers, la Terre, l'orientation dans l'espace, l'eau, le climat, le paysage, l'intervention de l'homme) ou la vie en société.

Outre les activités du livre-bilan, les élèves vont suivre le voyage du récit sur une grande affiche de classe, un planisphère qui leur sert à identifier d'abord les continents, les pays, puis les villes. En dehors de la classe, lors d'une randonnée, les élèves seront entraînés à savoir utiliser une boussole, par exemple. Ils feront un travail sur l'orientation dans l'espace (nord, sud, est, ouest) et sur la variété des paysages dans la nature (urbains, désertiques, forêts vierges, etc.). Le tour du monde en quatre-vingts jours permet également d'apprendre les différents moyens de transports (figure 5), aussi bien ceux qui sont nommés dans le récit que ceux que les enfants connaissent, selon que le voyage se réalise par terre ou par mer. Également l'œuvre permet d'examiner le monde qui entoure l'enfant profitant des voyages qu'ils ont réalisés aussi bien que de s'approcher de leurs pays d'origine. Cet aspect de l'œuvre fournit la possibilité de développer l'intégration et l'interculturel en classe. 
Fig. 5

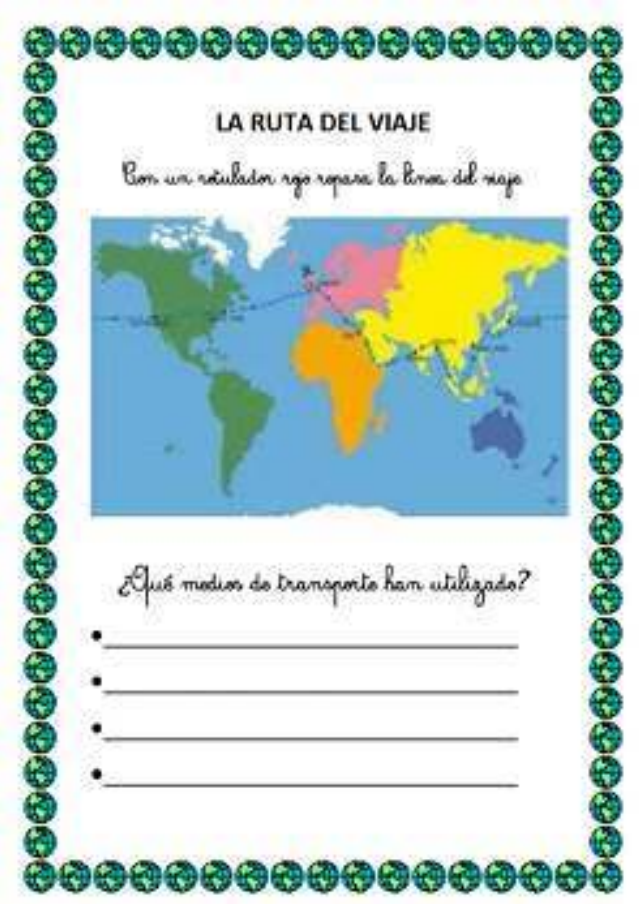

\section{Enseignement civique et social}

Selon la réglementation d'Aragon, le premier module de cette matière recueille les aspects sur l'identité et la dignité individuelle et renforce les relations interpersonnelles positives et enrichissantes, ainsi qu'une attitude contre la violence, les stéréotypes et les préjugés. Nous avons un exemple de cela dans les relations de respect entre les personnages de l'histoire, le refus de Fogg au sujet du destin d'Aouda en Inde ou dans le traitement des peuples (Hindous, Indiens, Européens, Orientaux) ainsi que des pays parcourus par les personnages et de leurs habitudes.

Le deuxième module porte sur la compréhension et le respect des relations interpersonnelles tout en cherchant le développement des habiletés sociales et communicatives, des bonnes relations avec les autres et aborde les valeurs sociales et civiques fondamentales telles que l'entente, la coopération, le pacte et le respect. Fogg en est un bon exemple parce qu'il observe les accords auxquels il est arrivé avec plusieurs personnages qu'il rencontre pendant son périple, pour atteindre la fin du voyage.

À travers les contenus du troisième module, on travaille sur les aspects de la cohabitation et les valeurs sociales. Le respect envers les idées et les croyances des autres y sont présents, par exemple quand les personnages entrent dans une pagode, fait qui permet de commenter les types de temples ou lieux de prière existants. Cet aspect facilite les approches à l'interculturel et aux différentes religions, très présentes dans les classes.

$\mathrm{Au}$ sein de cette matière, le programme scolaire comprend, entre autres aspects, la construction de l'identité personnelle, les relations interpersonnelles et l'attitude opposée à la violence. Nous retrouvons ici les relations de respect que maintiennent les personnages entre eux et le rejet de la violence exprimé par Fogg quand il délivre Aouda d'une mort certaine en Inde, lorsqu'elle va être tuée au nom d'une tradition ancestrale. 
Les élèves pourront comprendre l'interculturel, les traditions et la diversité des peuples des pays parcourus par les personnages ainsi que la tolérance à l'égard des différentes religions ; et partager tous les acquis avec leurs camarades (figure 6).

Fig. 6

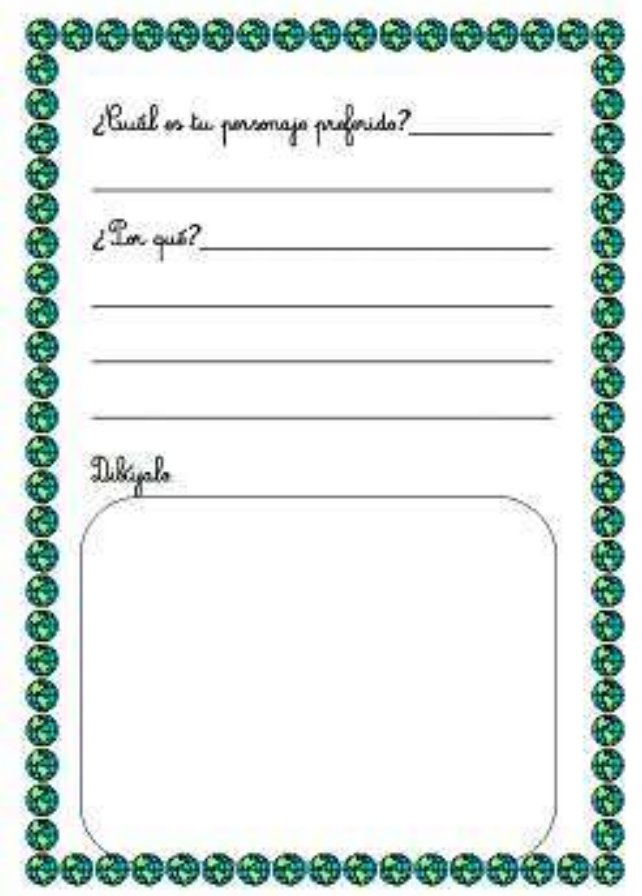

\section{Sciences de la nature}

Les contenus de cette matière se regroupent en plusieurs modules en rapport avec l'activité scientifique, les êtres vivants, l'être humain et la santé, la matière et l'énergie ainsi que la technologie, les objets et les machines.

Les différents pays que les personnages du roman traversent permettent de découvrir des êtres vivants : les animaux à plume et à poil, domestiques et sauvages, mammifères, oiseaux, reptiles, etc.

Les moyens de transports nous permettent de travailler sur les types d'énergie employée. Quant à la technologie et les machines, nous ferons allusion à d'autres romans de Verne, présents dans une activité du livre de l'élève que nous proposons. Il serait aussi possible aux élèves d'expérimenter dans la construction d'un bateau ou d'un sous-marin afin de vérifier la flottation ou l'immersion, selon le cas.

\section{Mathématiques}

Les Mathématiques s'identifient à la déduction, l'induction, la prévision, le rapprochement, la probabilité, la précision, la rigueur, la sécurité, etc. Elles permettent d'analyser des phénomènes et des situations qui se présentent dans le réel, d'obtenir des informations et des conclusions qui ne sont pas explicites, d'identifier des relations et des structures, de trouver des modèles, des régularités, des lois, pour finalement agir. Le travail par projets développe, particulièrement, ces habiletés chez les élèves. 
Le Programme s'organise autour des contenus étroitement liés et construits sur des apprentissages antérieurs et observables tels que les chiffres, la mesure, la géométrie ou la statistique et la probabilité.

Le roman permet, fréquemment, de construire des activités visant le calcul, par exemple pour compter le nombre de wagons du train, le nombre de saltimbanques du cirque, ainsi que l'argent que Passepartout va dépenser pour sa subsistance ou l'intendance du voyage, par exemple l'achat des vêtements d'Aouda. Il s'agit aussi de compter l'argent que Fogg va utiliser dans de nombreux échanges et transactions tout au long de son périple, afin de pouvoir poursuivre le voyage et gagner le pari dans les meilleures conditions.

\section{6. Éducation artistique}

28 En Espagne, la loi sur l'éducation (Lomce, 2013) considère l'art comme une activité qui apporte aux êtres humains un enrichissement personnel et intellectuel, qui va affermir des valeurs individuelles et collectives profondes, tout en développant des compétences créatives et imaginaires et qui situe les personnes ayant une formation artistique face à la sensibilité et à la beauté. L'Éducation artistique se divise en deux disciplines : Éducation plastique et Éducation musicale.

Dans les arts plastiques, l'éducation audiovisuelle fait référence à l'étude de l'image dans toutes ses manifestations, aussi bien visuelle qu'audiovisuelle. L'expression artistique envisage l'ensemble des concepts et processus qui ont été traditionnellement associés à cette discipline ainsi que le développement du graphisme et des savoirs acquis à partir du domaine des mathématiques dans la section de la Géométrie.

L'enseignant proposera de créer des dessins sur les personnages, les lieux, les paysages, les tempêtes, les animaux, etc., à partir des illustrations ou des descriptions minutieuses que Verne réalise. Pour cela, l'enseignant, ou un élève s'il sait le faire, lira des passages concrets aux enfants pour qu'ils imaginent cette représentation graphique. Une autre tâche consistera à faire colorier plusieurs dessins en utilisant techniques et styles différents : aquarelle, noir et blanc, abstrait, réaliste, cubiste, collage, etc. dans le but de faire à l'école une exposition sur le roman de Jules Verne.

L'éducation musicale met en place l'écoute en relation avec la perception et elle inclut l'ensemble des contenus en relation avec l'exploration, la reconnaissance et la valorisation $\mathrm{du}$ son selon ses possibilités expressives. Elle comprend aussi le développement de connaissances et habiletés techniques vocales et instrumentales appliquées à la création et à l'interprétation musicales. Finalement, le mouvement et la danse englobent l'action corporelle en tant que source, instrument et condition fondamentale de la connaissance. L'Éducation musicale fait référence au travail du corps comme moyen d'intériorisation et d'expression des éléments musicaux.

Pour favoriser la reconnaissance des sons, les techniques vocales et l'interprétation musicale, au commencement de chaque séance consacrée à la réalisation du projet, l'enseignant fera écouter attentivement la musique qui identifie Le tour du monde et les élèves devront chanter les paroles 5 .

\section{Education physique}

L'Éducation physique contribue au développement de l'individu dans sa totalité, ce qui implique des aspects moteurs, biologiques, cognitifs, émotionnels et de relation 
interpersonnelle. Cette discipline favorise l'activité physique orientée à la participation autonome et satisfaisante tout au long de la vie. Les contenus sont en rapport avec les valeurs de la vie active, les actions motrices individuelles, d'opposition, de coopération, en milieu naturel ou artistiques et expressives.

Les personnages de Jules Verne dans Le tour du monde en quatre-vingts jours sont aussi des exemples liés aux contenus de cette matière puisqu'ils bougent et se déplacent constamment, ils ont besoin de la coopération pour aboutir à leur destin, ils opèrent dans des milieux naturels. Phileas Fogg, parce qu'il voyage, est un modèle de vie active.

\section{Considérations finales}

31 L'élaboration de ce travail nous amène premièrement à collaborer avec des professeurs des écoles dont les contributions fondamentales et non négligeables servent à cibler les activités que nous proposons, dans le niveau correspondant. L'approche du roman de Jules Verne nous permet de présenter un travail sur la langue (la syntaxe et la grammaire), mais aussi sur la communication favorisant, ainsi, les liens entre les membres de la classe et la dimension interculturelle, et facilitant son perfectionnement, sans faire une "classe de langue ", proprement dite : les enfants sont invités à parler constamment et à s'exprimer pour développer leur langage, leur pensée et par conséquent pour mieux comprendre le monde qui les entoure.

Cette compétence langagière ainsi que la pédagogie par projets, liée au domaine de la motivation pour la réalisation des tâches, vont faire place, indirectement, à la géographie, à l'histoire, aux sciences, aux arts, etc., et vont permettre un apprentissage plus significatif aux plus jeunes apprenants.

Ce que nous venons d'exposer est la présentation d'un travail que nous sommes en train de produire. Il reste encore à faire une tâche considérable avant d'avoir tous les éléments nécessaires (livres, fiches, textes, etc.) qui permettront de familiariser les élèves avec Jules Verne.

\section{BIBLIOGRAPHIE}

ABDALLAH-PRETCEILLE, Martine \& PORCHER, Louis (1996). Éducation et communication interculturelle. Paris : Presses universitaires de France, collection Éducateur.

AlLAM-IDDOU, Samira (2015). « De l'usage des textes littéraires comme outil didactique pour l'enseignement/apprentissage du Français Langue Étrangère ", Synergies Chili, nº11, pp. 95-103. ALONSO TAPIA, Jesús (1997). Motivar para el aprendizaje. Teoría y estrategias. España : Ed. Edebé. BORDALO, Isabelle \& GINETEST, Jean Paul (2006). Pour une pédagogie du projet. Paris : Hachette Éducation.

CITTON, Yves (2007). Lire, interpréter, actualiser. Pourquoi les études littéraires ? Paris : Éditions Amsterdam. 
FERREIRO, Emilia \& TEBEROSKY, Ana (2005). Los sistemas de escritura en el desarrollo del niño. Madrid: Siglo XXI Editores S.A.

LARMER, John \& MERGENDOLLER, John R. (2010). The Main Course, Not Dessert. Buck Institut for Education. [online] <URL: http://www.bie.org/object/document/main_course_not_dessert> OLIVER, Esther \& GATT, Suzanne (2010). « De los actos comunicativos de poder a los actos comunicativos dialógicos en las aulas organizadas en grupos interactivos ", Revista Signos, nº43 (2), pp. 279-294.

PROULX, Jean (2008). Apprentissage par projet. Québec : Presses de l'Université du Québec. Collection Formules pédagogiques.

RESCOL (RÉSEAU SCOLAIRE CANADIEN) (1998). Apprendre ensemble par projet avec l'ordinateur en réseau. Guide à l'intention des enseignants et des enseignantes. (actualisé le 31/05/2001) <URL : http:// www.tact.fse.ulaval.ca/fr/html/sites/guidep.html> SÉoud, Amor (1997). Pour une didactique de la littérature. Paris : Hatier/Didier, collection LAL. TEBEROSKY, Ana \& FERREIRO, Emilia (2000). Sistemas de escritura, constructivismo y educación. Rosario : Homosapiens Ediciones.

TEBEROSKY, Ana (1990). «El lenguaje escrito y la alfabetización », Lectura y vida. Revista latinoamericana de lectura, ํㅡㄴ, pp. 2-15.

TEBEROSKY, Ana (2003). « Primeras escrituras en segundas lenguas y contexto multilingüe » in Soler, Marta y Teberosky, Ana (Coords.). Contextos de alfabetización inicial. Barcelona : Horsori. TEBEROSKY, Ana \& COLOMER, Teresa (2001). Proposta constructivista per apprendre a llegar i a escriure. Barcelona : Vicens Vives.

\section{Espagne : Réglementation nationale sur l'Éducation}

LOMCE, 2013 : Ley Orgánica 8/2013, de 9 de diciembre, para la mejora de la calidad educativa. Boletín Oficial del Estado no $295,10 / 12 / 2013$.

Real Decreto 126/2014, de 28 de febrero, por el que se establece el currículo básico de la Educación Primaria. Boletín Oficial del Estado no 52, 01/03/2014.

\section{Aragon : Réglementation autonomique sur l'Éducation (Arrêtés)}

ORDEN de 16 de junio de 2014, de la Consejera de Educación, Universidad, Cultura y Deporte, por la que se aprueba el currículo de la Educación Primaria y se autoriza su aplicación en los centros docentes de la Comunidad Autónoma de Aragón. Zaragoza : Boletín Oficial de Aragón, no 119, 20/06/2014.

ORDEN de 28 de marzo de 2008, del Departamento de Educación, Cultura y Deporte, por la que se aprueba el currículo de la Educación Infantil y se autoriza su aplicación en los centros docentes de la Comunidad Autónoma de Aragón. Zaragoza : Boletín Oficial de Aragón, nº 43, 14/04/2008.

\section{NOTES}

1. Le projet s'insère dans le contexte éducatif espagnol dont il suit la réglementation ainsi que les normes spécifiques qui régissent en Aragon.

2. Voir à ce sujet Yves Citton, 2007. 
3. VERNE, Jules (1873). Le tour du monde en quatre-vingts jours. Illus. Neuville. Paris : J. Hetzel.

4. Voir note 5.

5. La musique et les paroles sont disponibles sur Internet: https://www.youtube.com/watch?

$\mathrm{v}=7$ wai7_miiEo

\section{RÉSUMÉS}

Nous proposons dans ce travail un projet à développer en Aragon (Espagne) dans les écoles au niveau de la Maternelle et du Primaire, qui va rapprocher Jules Verne aux enfants d'une manière motivante, tout en respectant les domaines d'apprentissage propres à chaque niveau. Pour cela nous avons choisi le roman Le tour du monde en quatre-vingt jours. Nous considérons d'une part qu'il est avantageux de mettre en place la méthode d'apprentissage par projet puisqu'elle permet de travailler différentes stratégies d'apprentissages, de placer l'élève au cœur de son parcours scolaire en réalisant des tâches significatives pour lui et de mener un travail de collaboration entre tous les membres de l'école. D'autre part, le travail sur ce texte adapté nous permet une approche communicative à travers la langue, dont l'enfant a besoin pour son épanouissement intellectuel et pour développer son esprit critique. Nous présentons uniquement quelques activités en tant que démonstration d'un projet envisagé pour l'année scolaire complète et auquel participent tous les élèves.

In this work, we propose a project to be developed in Aragon (Spain) in schools at Nursery school and Primary sector levels, which approaches Jules Verne to the children in a motivational way, respecting the appropriate areas of learning in each level. For it we have chosen The world tour in eighty days novel. On one hand, we consider that setting up the methodology of learning by projects is advantageous because it allows working in various learning strategies, placing the pupil at the core of his school route by performing significant tasks for him and leading a work of collaboration among all the school members. On the other hand, working on this text brings us a communicative approach through the language, which is required by the child for his intellectual growing and developing his critical eye. We have just created some activities as a demonstration of a project that is planned for the whole school year and involves the participation of all the pupils.

\section{INDEX}

Mots-clés : Verne (Jules), apprentissage par projet, école en Espagne, motivation, tâche

Keywords : Verne (Jules), learning project-based, school in Spain, motivation, task

\section{AUTEURS}

\section{MARÍA-TERESA BAREA}

Université de Saragosse

tbarea[at]unizar.es 


\section{MARÍA-LOURDES CADENA}

Université de Saragosse

mlcadena[at]unizar.es

\section{ANA-MARÍA CLAVER}

Université de Saragosse

aclaver[at]unizar.es

MARÍA-PÍLAR TRESACO

Université de Saragosse

ptresaco[at]unizar.es 